\title{
Germanica
}

les arts dans les pays germanophones au $\mathrm{XX}^{\mathrm{e}}$ siècle

\section{Benn und das fünfte Jahrhundert}

Benn and the fifth century

Benn et le cinquième siècle

\section{Holger Schmid}

\section{OpenEdition \\ Journals}

Édition électronique

URL : http://journals.openedition.org/germanica/813

DOI : 10.4000/germanica.813

ISSN : 2107-0784

Éditeur

Université de Lille

Édition imprimée

Date de publication : 30 décembre 2009

Pagination : 44-58

ISBN : 978-2-913857-24-7

ISSN : 0984-2632

\section{Référence électronique}

Holger Schmid, «Benn und das fünfte Jahrhundert », Germanica [En ligne], 45 | 2009, mis en ligne le 01 décembre 2011, consulté le 06 octobre 2020. URL : http://journals.openedition.org/germanica/813 ; DOI : https://doi.org/10.4000/germanica.813

Ce document a été généré automatiquement le 6 octobre 2020.

(C) Tous droits réservés 


\title{
Benn und das fünfte Jahrhundert
}

\author{
Benn and the fifth century \\ Benn et le cinquième siècle
}

Holger Schmid

1 „Eigentlich hätte ja mit den Griechen Schluß sein können”, schreibt Gottfried Benn einmal in einem Brief. Ist es der Grundaffekt deutscher Griechenland-Sehnsucht seit Winckelmann, welcher so, mitten in den Verwerfungen des 20. Jahrhunderts, mit allem Glanz und Elend aufersteht? Soll man ein lyrisches Werk wie den Zyklus dreier Gedichte, mit dem Titel V. Jahrhundert, betrachten als ein Zeugnis solches gleichsam unverbesserlichen Philhellenismus - mit allen bekannten Nachteilen: Ablenkung von der Gegenwart, besonders auch der politischen des Dritten Reichs, Verrat der avantgardistischen Anfänge des Expressionisten, Haß auf die Gegenwart der Moderne, Weltflucht zugunsten der Hinwendung zu einer idealisierten Perikles-Zeit, kurz als Inbegriff der nostalgie de la Grèce, wenn nicht der tyranny of Greece over Germany? Könnte man selbst derartige Zeichen aus den Texten erheben, wäre dann auch der Poesie schon Recht geschehen?

2 Im Streit um den Modernitäts-Charakter der Dichtung des 20. Jahrhunderts sieht man zumeist den Bezug zur Antike nicht im Vordergrund, erst recht nicht als ein gattungsspezifisches Element. Was davon aufscheint, sind gemeinhin Fragen der Einfühlung in antike Stoffe und Motive: Leda bei Yeats (1918), Ulysses und Vergil bei Joyce und Broch, Edipus bei Cocteau - also so etwas wie die Aktualität dieser nacherzählten Geschichten, sofern sich in ihnen moderne Wirklichkeit „noch” (wie man, zuversichtlich oder skeptisch, sagt) zur Sprache bringen läßt. Weniger, und schon kaum für die Lyrik, steht das Verhältnis zur archaischen Welt als Reflexion auf Geschichte und Vergangenheit im Blickpunkt. Dies ist aber Benns Fall, der sich an vorderster Front an jenem Streit beteiligt, und der sich speziell im Blick auf seinen Fehltritt von 1933 den doppelten Vorwurf des Irrationalismus und der RealitätsVerweigerung zugezogen hat und zuzieht. ${ }^{1}$ Hier genau kreuzen sich mithin die Probleme des politischen Engagements (soweit davon die Rede sein kann) und der poetischen Modernität. 
3 So mutet Benn, der in Deutschland gebliebene Dichter von Rang, wie ein exemplarischer Fall an: die konsequent aus der Ich-Zerstörung der expressionistischen Anfänge entwickelte, in den großen Gedichten der zwanziger Jahre zum dionysischrauschhaften Hinsinken gesteigerte Intellektfeindschaft und die darin implizierte Antimodernität scheinen geradewegs an Hitlers Seite zu führen. Die in der Vergötzung der "Macht” gipfelnde, doch von weit her kommende Ablehnung der Vernunft (der mit dem "griechischen Wunder”, der naissance de la raison, zur Welt gelangten Ratio,) ebnet den niederen Dämonen den Weg. So lautet die wohl überwiegend vertretene Ansicht; außer Zweifel steht, daß hier ideologische Gefühle und künstlerische Strukturen direkt ineinander liegen (und davon ist zu reden, wenn man mit einem Gedicht von $1944 \mathrm{zu}$ schaffen hat).

Sieht man näher zu, beginnen hier aber auch diffizile Fragen. Die Absage an das neuzeitliche „Ich”, wie sie der magisch-entrückt strömenden Lyrik Benns in den zwanziger Jahren zugrunde liegt (sie trägt den für die ganze Epoche konstitutiven Widerstreit von Leben und Geist aus, wie ihn besonders seit 1900 das Denken der Brüder Mann, später notorisch die Weltanschauung Ludwig Klages' offenbart), begünstigt also jenen Irrationalismus, den man als die gerade Bahn zu Hitler aufzufassen gewohnt ist: so hat es dann auch, nach Thomas Manns Wendung zur Republik, die Pariser Rechenschaft von 1926 einflußreich dargestellt. Die einseitige Hingabe an das Rauschhaft-Dionysische und Feindschaft gegen das Geistige wird beantwortet durch ein Bekenntnis zum Apollinischen und Anti-Dionysischen, also zu „Maß und Vernunft” gemäß den Werten der Aufklärung. Exemplarisch beruft sich Mann zu allen Zeiten auf eine analoge Wende in der Nietzsche zugeschriebenen Überwindung der Romantik; da so indes zugleich Benns lebenslanges Leitbild in Frage steht, läßt sich der Konflikt sehr wohl als ein Streit um Nietzsches Vermächtnis ansehen. Der Poesie stünde dergestalt eine ähnliche Selbstreform bevor, wie sie, in der Überwindung des irrationalistischen „Sturm und Drang” hin auf den Humanismus der Weimarer Klassik, Goethe und Schiller geleistet hatten. Ein solches Postulat steht nun wiederum in Parallele zu Gedankengängen um eine „Klassik II” als Frucht des modernen Expressionismus, präzise als Überwindung jener irrationalistischen Auflösung durch einen Kult der Form, wie sie Benn seinerseits aus Nietzsche und ebenso den Frühwerken Stefan Georges und Heinrich Manns abzuleiten pflegte. Daß es somit analog zu Weimar, mutatis mutandis, um den Bezug zur Antike gehen könne oder müsse, drängt sich schon auf, auch wenn man in diesem Zusammenhang das über die Kulturen des 20. Jahrhunderts verbreitete Phänomen der Klassizismen noch gar nicht ins Auge gefaßt hätte. In Benns Denken selbst haben gelehrte Ausleger ihrerseits eine entsprechende Entwicklung von jenem Rauschhaft-Geistfeindlichen zum NüchternAbgeklärten angenommen, wonach also - gerade mit der Krisis von 1933-34 einsetzend - eine sozusagen vergeistigende Wendung zum „statischen”, im Zeichen der „Pallas” stehenden Kosmos der späteren Werke als Quintessenz von Benns poetischer Geschichte vorliegt. ${ }^{2}$ Wie sich versteht, erscheint somit das Verhältnis zur Antike 1944 an entscheidender Stelle; und im besonderen stellt sich die Frage nach dem fünften Jahrhundert. 
Indessen komplizieren sich dabei die Dinge nicht unbeträchtlich. Der apollinische MaßGedanke, insofern er jenem Rauschhaft-Dionysischen entgegengestellt wird, bildet nämlich in Benns Zusammenhang nichts anderes als die Idee des „Dorischen”, in deren Namen die jähe ideologische Wendung von 1933 steht: also die Parteinahme für die "Macht” und die „Züchtung”, zu Gunsten des neuen Staates. Von hier aus wird umgekehrt derselbe romantisch-musikalische Überschwang, den seinem eigenen Frühwerk Nietzsche in beredten Worten vorgeworfen hatte, als ein Obsoletes bekämpft. Diametral entgegengesetzt zur Konzeption des Griechentums aus der Idee eines dionysischen Grundes, dem regenbogengleich die glanzvolle Vision des Apollinischen entspringt, wird die "dorische Welt”, verkörpert durch Sparta das „Männerlager am Eurotas”, ganz ins Maß Apollons gestellt: „Wir leiten also aus Sparta Griechenland ab, und aus dem Dorisch-Apollinischen die griechische Welt. Dionysos steht hier wieder in den Grenzen, in denen er vor 1871 („Geburt der Tragödie aus der Musik”) stand.” Hieraus begreift also Benns Essay den griechischen Geist und die Welt des fünften Jahrhunderts. Die Bestimmung des Dorischen als Grund des Griechentums hat der Dichter übrigens, ablesbar an den Wiederveröffentlichungen des Textes nach 1945, nicht erkennbar revidiert: der Intellekt - verkörpert durch „Pallas”, die waffenstarrend jungfräuliche Athena - bleibt leitende Forderung, die Ablehnung von Geschichte im Namen des Entwicklungsfremden entspricht dem vollkommen. ${ }^{4}$ Es läßt sich daher mit Zuversicht erwarten, eine just zur selben Zeit wie der Pallas-Essay entstandene, den Titel V. Jahrhundert tragende Dichtung werde rein im Zeichen des Dorisch-Palladischen stehen und dergestalt Benns Verhältnis zum Griechentum exemplarisch ausdrücken.

6 Wer daraufhin den aus drei Gedichten bestehenden Zyklus dieses Titels zur Hand nimmt, mag freilich überrascht sein: es findet sich eben von jenem Dorischen darin keine Spur. ${ }^{5}$ Auf allgemeiner Ebene wird man dadurch an die grundsätzliche Warnung erinnert, sich Kriterien und Begriffe der Gebilde-Erfahrung nicht durch die Selbstinterpretation eines Autors aufzwingen zu lassen. Ob freilich über NichtKongruenz (falls sie zutrifft) hinaus mehr vorliegt, etwa signifikante Widersprüche, bleibt die Frage; nur auf Grund genauerer Wahrnehmung der Gedichte wird sie zu ermessen sein.

7 Früher als die eben erwähnte, erst dem Nachdenken auffällige Beobachtung drängt sich wohl die Frage des Zusammenhangs der Teile auf: Was verbindet die Elemente? Findet sich eine einheitstiftende Eigenschaft, die den „Zyklus” rechtfertigt? Gewiß handelt es sich nicht um ein thematisch durch Charakter oder Intrigue zusammengehaltenes Werk (wie z. B. die Nietzsche-Suite Sils-Maria ${ }^{6}$ ). Die Dinge hängen zunächst gerade nicht zusammen; so denkt man an die von Benn erhobene Grundforderung des „Verharrens vor dem Unvereinbaren", Konstituens poetischer Modernität zugleich mit der nihilistischen Anschauung von „Leben”, Welt und Geschichte. Doch bedenkt man näherhin, daß sich im Innern der Teile gerade dieselbe Frage wiederholt, so erkennt man auch die Anknüpfung an jenem Zerbrechen der Synthesen und des Subjekts, das mit der Zerstörung des „Ich” verbunden war. Wir haben es also mit einer deutlichen Beziehung auf die expressionistischen Grundlagen von Benns Poetologie und ebenso auf die Melodik der lyrisch sich verströmenden Dichtungen der zwanziger Jahre zu tun. Das ist keine Entdeckung: allgemein ist schon für die vierziger Jahre ein gewisses 
Aufleben früherer Sprachhaltungen (eben das Dionysische der Jahre vor 1930, zum "Orphischen" geläutert) wahrgenommen worden. ${ }^{7}$ Es wiederholt sich aber von daher, und unter den gleichsam verschärften Umständen der „dorischen Welt”, die Frage des Bezuges zwischen dem rauschhaft-hinsinkenden Melos und der Strenge der strophischen Fügung, aus welcher sich eine poetische Einheit konstituiert, die seit der Erneuerung der Dichtersprache durch Mallarmé und Rimbaud nicht mehr die der semantischen Linearität der Zeichen sein kann. Anders ausgedrückt, es stellt sich konkret und präzise am Werk selber das Problem der Selbständigkeit der Teile, und das heißt zugleich: der Natur des „Ganzen” eindringlicher dar. In der Art, wie die Epoche des „Statischen” zu der singenden Sprache der früheren Epoche zurückhorcht, wird demnach das bedingt liegen, was Benn zum fünften Jahrhundert sagen will.

$8 \quad$ Fast wie ein Inbegriff der geschilderten Problematik wirkt das Beispiel des Gedichtes Welle der Nacht, dessen zweite und letzte Strophe nach Mitteilung des Dichters (rund) zwanzig Jahre nach der ersten entstand, und zwar 1942-43. ${ }^{8}$

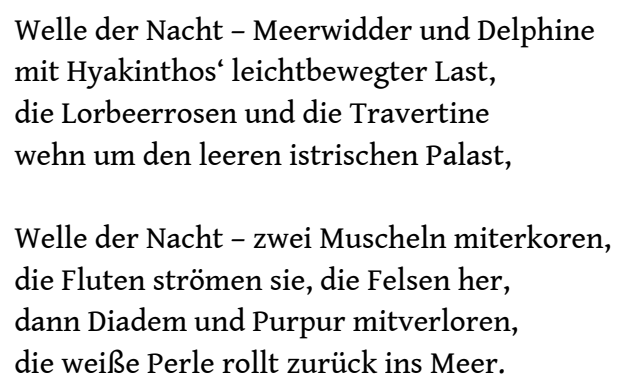

Was war an der wunderbar gelungenen Fügung, die jenes Melos der früheren Epoche bruchlos aufnimmt, so schwer zu finden? Beschränken wir uns hier auf die Erwägung, daß es nicht der Stoff war, der laut dem Essay Kunst und Staat schon 1927 (also vermutlich bei Entstehung der Anfangsstrophe) dem Dichter gegenwärtig war: der istrische Palast des Kaisers Diokletian in Spoleto, das Diadem, der Purpur, das Unaufhörliche des Meeres (auch die Muscheln sind irgendwo), die „weiße Perle”. ${ }^{9}$ Das heraklitische Wogengefühl, nicht anders als die Wort-Materie, ist vorhanden: allein das Passen selber, die „Harmonie”, stand aus. Es muß so etwas wie der von Benn gleichnishaft beschworene Schlag des Glasbläsers gewesen sein, mit dem plötzlich die Elemente zusammentraten, und vor dem wir also mit unserer Frage nach der Einheit des Griechen-Zyklus wieder stehen.

10 Um auf die „Welt des fünften Jahrhunderts” und jenes Nicht-Dorische zurückzukommen, so liegen auch hier auf der Ebene des Stofflichen die Dinge einigermaßen einfach: für seinen Zyklus versieht sich Benn fast ausschließlich aus einer einzigen Quelle, welcher er den größten Teil seiner Motive, oft beinah wortgetreu, entnimmt. Es ist Psyche, das bekannte Werk über griechischen Seelenkult und Unsterblichkeitsglauben von Nietzsches Freund Erwin Rohde. ${ }^{10}$ Nichts vom Dorisch-Apollinischen: die Sphäre einer unheroischen Begräbnisfeier und Totenklage, schroff danebengesetzt eine Art Stiftungslegende der Mysterien von Eleusis, zuletzt gerade so unvermittelt das Aufrufen entrückter Schatten der Vorzeit. Als sichtbarer Leitfaden bietet sich das Thema von Tod und Unsterblichkeit - aber liegt nicht schon darin ein Irrtum? Von einer Bewältigung des Todesproblems hat man gesprochen: es 
handle sich um so etwas wie einen Ascensus, ausgehend von der Trauer und letzten metaphysischen Einsamkeit des Individuums im Augenblick des Sterbens, dann aber die im eleusinischen Geschehen liegende Verheißung, wie sie zur Seligkeit, ja Unsterblichkeit führt. ${ }^{11}$ Von der Klage über die Vergänglichkeit zum Triumph über den Tod - es wäre eine Schillersche Bewegung, die vor allem durch das zum Schluß genannte Moment des „Spieles” bekräftigt scheint. Ist dies die Struktureinheit von klassischer und moderner Dichtung? Ist Benn, der radikale Moderne, hier zugleich ein Nachfahr des klassischen Weimar?

V. Jahrhundert

I

„Und einer nimmt die attische Lekythe, auf der die Überfahrt von Schlaf und Staub in weißen Grund gemalt als Hadesmythe, zwischen die Myrte und das Pappellaub.

Und einer steckt Zypresse an die Pfosten der lieben Tür, mit Rosen oft behängt, nun weißer Thymian, Tarant und Dosten den letztesmal Gekränzten unterfängt:

Das Mahl. Der Weiheguß. Die Räucherschwaden. Dann wird ein Hain gepflanzt das Grab umziehn und eine Flöte klingt von den Zykladen, doch keiner folgt mir in die Plutonien."

II

Das Tal stand silbern in Olivenzweigen, dazwischen war es von Magnolien weiß, doch alles trug sich schwer in Schicksalsschweigen, sie blühten marmorn, doch es fror sie leis.

Die Felder rauh, die Herden ungesegnet, Kore geraubt und Demeter verirrt, bis sich die beiden Göttinnen begegnen am schwarzen Felsen und Eleusis wird.

Nun glüht sich in das Land die ferne Küste, du gehst im Zuge, jedes Schicksal ruht, glühst und zerreißest dich, du bist der Myste und alte Dinge öffnen dir dein Blut.

III

Leuké - die weiße Insel des Achill!

Bisweilen hört man ihn den Päan singen, Vögel mit den vom Meer benetzten Schwingen streifen die Tempelwand, sonst ist es still.

Anlandende versinken oft im Traum.

Dann sehn sie ihn, er hat wohl viel vergessen, er gibt ein Zeichen, zwischen den Zypressen, 
weiße Zypresse ist der Hadesbaum.

Wer landet, muß vor Nacht zurück aufs Meer.

Nur Helena bleibt manchmal mit den Tauben,

dann spielen sie, an Schatten nicht zu glauben:

„- Paris gab dem den Pfeil, den Apfel der - "12

Der erste Abschnitt also, eingefaßt in die rätselhaften Bennschen Anführungszeichen, bildet die Evokation einer attischen Bestattungszeremonie, markiert durch die weißgrundige Lekythos (mit ihren schwarzen Zeichnungen, hier vielleicht der CharonsNachen) und den ganz beherrschend im Vordergrund stehenden Pflanzenschmuck. Es stammen, wie gesagt, die stofflichen Einzelheiten des Kultes aus Rohde (mit Ausnahme der Zykladen, dort ist von karisch-phrygischen, also ausdrücklich orgiastischen Flöten die Rede): die auf die Unterwelt verweisenden Gewächse Myrte, Zypresse und Pappel (die von Herakles aus dem Hades gebrachte Silberpappel?), die das Haus des Toten bezeichnen und verwandeln; Doste und Tarant, auf die er gebettet wird. Dazu fügt sich die weiße Farbe, die von Trauer und Tod: der Grund des Grabgefäßes, der Thymian und die Myrte, wohl auch die Zypresse und die Pappel, deren griechischer Name „Leuké” ist. Kontrapunktisch dagegen, im Modus der Erinnerung, erscheint die Rose als Sphäre der lebendig-erotischen Schönheit - und wir beginnen um das Weiß ein Spiel der Farben zu ahnen, das angesichts von Benns berüchtigtem Spott über die Farben als lyrische Elemente verwundern könnte. Bei dem Mahl der dritten Strophe legt sich die Annahme nahe, als Empfänger des Weihegusses sei der im Grab noch anwesende Tote selbst als Teilnehmer, oder eher noch als Gastgeber der Veranstaltung gedacht: so würden sich auch die Anführungszeichen wie die Rede in der ersten Person verstehen lassen. Die gemalte „Überfahrt” des Anfangs scheint dieselbe zu sein wie der zuletzt angedeutete Gang zu den Hadestoren, den „Plutonien” ${ }^{13}$; ein eigentümlicher Raum von Gleichzeitigkeit entsteht durch die substantivische, nicht-erzählende Sprache des Gedichts.

Der zweite Teil des Zyklus beginnt hingegen im Vergangenheitstempus mit der Evokation der vom Frost überhauchten Frühlingslandschaft des „Tales”, die dem Zustand der Abwesenheit der entführten Kore in der Unterwelt zu entsprechen scheint; die zweite Strophe könnte noch dazugehören, sofern man deren einziges Verbum "wird” als ein episches Präsens auffaßt. Andererseits ergibt sich bei dem „bis”, genau in der Mitte des Zyklus, ein Umschwung ins Gegenwärtige, bestärkt durch das folgende „nun”: es ist also der Augenblick der Anodos, Kores Rückkehr ans Licht, rhythmisch in der Gegenbewegung zum Gang in die Plutonien. Mit ihm verknüpft sich in der hieratischen Geschichte die Hoffnung auf ein schöneres Schicksal nach dem Tode, wie es der homerische Demeter-Hymnus den Eingeweihten verspricht, also durch „Eleusis”. Hier hat Benn zu den Nachrichten seiner Quellen den "schwarzen Felsen" hinzuerfunden: es ist bemerkenswert, wie dadurch nun das spezifisch Dichterische dieses Abschnitts, das Gleichgewicht zu dem Weiß und Silber von Magnolien und Oliven, in der Begegnung der Göttinnen hervorgebracht wird. So entstehen aber auch, zurückhörend in den ersten Abschnitt, neue Schwingungen des Sinns: die weiße Pappel, Leuké, mit der dunkel-hellen Doppelfärbung ihrer Blätter, welche die Griechen auf den Zusammenhang von Ober- und Unterwelt verweist; der Zusammenklang von Rosen und Myrten (auch diese zu beiden Sphären gehörig), den Blumen der Aphrodite, in eigentümlicher Kontrapunktik auf Weiteres vorbereitend. 

überraschen. Nicht die Jenseits-Verheißung, sondern das Moment des Gehens „im Zuge" steht im Blick: der sich selber anredende Myste befindet sich nicht im Telesterion, am Gipfel der stummen, verzückten Schau, mit der gerade die Aussicht auf ein seliges Schicksal verbunden war; vielmehr bewegt er sich in jenem Glühen und „Sich-Zerreißen” einer von Benn als „phrygisch” bezeichneten Kommunion. Es liegen mithin Korrelate vor zu der „sein Inneres mit Worten zerreißenden”, das kausalhistorische Ich-Konzept halluzinatorisch durchstoßenden Kondition des modernen Dichters. Soweit also das „du” zugleich das poetische Subjekt ist, hätte man es bei solcher Eröffnung alter Dinge nicht mit einer der geläufigen, „poetologischen” Selbstallegorisierungen des Schaffensprozesses zu tun, sondern vielmehr mit einem Beispiel der Realisierung jener von Benn häufig dargestellten, von ihm als "geologisch” aufgefaßten Ich-Struktur, die sich keineswegs von ungefähr am Moment der zyklischen Wiederkehr der Kore entzündet, am Auf und Ab des Unaufhörlichen: der Weg hinauf und hinab ist derselbe. Wann ist also das „Nun” des Mysten? Es ist dieselbe Frage wie die andere, was der Dichter aus seinen Rohde-Exzerpten eigentlich gemacht habe. Oder wiederum anders ausgedrückt, es ist die Frage des Zusammenhangs der Teile im Werk: verschärft diesmal, denn abrupt geht nun offenbar der folgende Abschnitt des Zyklus in eine ganz andere Sphäre über (nachdem das Bisherige, zumindest durch die Linie von „attisch” zu „Eleusis”, thematisch noch irgendwie zusammengehalten wirkt).

Das dritte und abschließende Gedicht beschwört den Peliden Achilleus, von dem gewisse Überlieferungen sagten, er sei von seiner Mutter Thetis, nach seinem durch die Hand Apollons und Paris' bewirkten Tod vor Troia, auf eine ferne selige Insel entrückt worden. Man identifizierte diese „Leuké” mit einer Insel an der Donaumündung, wo auch Achilleus einen Kult erhielt; doch Rohde sah in ihr den ursprünglich rein mythischen Ort einer Silberpappel („Leuké”) am Hades-Eingang. Die „weiße Insel” in ihrer Stille, auch mit ihren Zypressen an den ersten Abschnitt als Echo der „Plutonien” erinnernd, bildet den Raum des entrückten Achill, wie er in seiner musikalisch (im Päan) und mantisch (im Weissagen) ganz durch das Apollinische bestimmten Existenz dort währt. Das Traum-Bild, als welches er den Schiffern erscheint, verstärkt übrigens den Gedanken an die Kennzeichnung der apollinischen Sphäre in Nietzsches Geburt der Tragödie. Sofern sich aber dieses Dasein als Entelechie des Irdischen darstellt (und zugleich im Einklang mit der bei Benn selbst zu beobachtenden Entwicklung hin zum Apollinischen, von der vorhin die Rede war), mag es naheliegen, bei dieser Vergegenwärtigung Achills so etwas wie einen Durchbruch des Menschlichen zur Transzendenz zu erblicken, vergleichbar dem Aufstieg des Herakles zum Olymp in Schillers Reich der Schatten: trostvolle Verheißung der Unsterblichkeit für den Menschen schlechthin. ${ }^{14}$

Ort steht indessen im Zeichen des „Hadesbaums”, der weißen Zypresse; auch als Raum des Vergessens, von dem Achill gezeichnet ist, gemahnt er an den Hadeseingang, wo sich nach der Tradition die Lethe, die Quelle des Vergessens befindet. Achill ist so auch nichts anderes als ein „Schatten”, der den Lebenden im Traum nur sichtbar wird: er teilt sich nicht in menschlicher Weise ihnen mit, sondern wie der Herr von Delphi, welcher seinen Spruch (wie man das heraklitische Wort seit Plutarch versteht) „andeutet". ${ }^{15}$

16

Doch diese Licht- und Tageswelt, in der nur der Traum der Schiffer einziehen kann, ist lediglich ein Teilaspekt dieser Existenz. Es gibt sodann den Gegensatz, welchen, ein 
Wunder an Dichte und Tektonik, die letzte Strophe des Gedichtes bringt. Die Nacht ist der Raum einer Gegenwelt aphrodisisch-erotischer Schönheit, in dem sich „manchmal” die Epiphanie der gleichfalls entrückt geglaubten Helena ereignet. ${ }^{16}$ Sie kommt geleitet von den Tauben, die nun den Myrten des ersten Teils antworten: in der Tat zeigen sich diese hier, im Zurückhören, als umschlagend in Friedens- und Glückssymbole im Zeichen der Aphrodite. Das heißt, der Raum der Insel („die Leuchtende” nennt sie Pindar $)^{17}$ ist selbst wiederum durchwirkt von jenem Ineinander oder Wechsel von Schwarz und Weiß, in dem das Spiel der beiden „Schatten" stattfindet: das Umschlagen von Leben und Tod, wie es die Dichtung von ihrer Mitte aus erblickt, wird die Frage nach dessen Charakter erlauben.

Es ist nun Benns Gedanke, der aus dem Topos der Vereinigung von Achill und Helena das Element des Spiels aus der gemeinsamen Beziehung zu der Gestalt des Paris herausholt: der Bogen des Kämpfers, dessen sich Apollon zur Tötung Achills bedient; das Schönheits-Urteil zu Gunsten der Aphrodite, das zum ganzen Krieg um Troja führte. Als Gegensatz zu Traum und „Vergessen” des Tages ist nun aber diese nächtliche Sphäre des Spiels gerade eine der Erinnerung selbst, unter Schatten: aufzufinden bleibt also die Kunst-Reflexion, die auf „Schein” und Schönheit. Was läßt sich demnach, in aller Knappheit und Eile, über das Verhältnis zu Schiller ausmachen?

Die Sphäre von Benns Spiel zerfällt bei Schiller in zwei getrennte Phänomene: Auf der einen Seite findet sich das Spiel, in dem allein der Mensch ganz Mensch ist - denn das heißt in der oft übersehenen Schillerschen Konsequenz: nur Mensch, dem es bestimmt ist, im Ausgang von solchem „Morgentor” sein erst im moralischen Zustand liegendes Ziel unausgesetzt zu erstreben. Auf der anderen Seite steht das symbolische Bild dieser moralischen Vollendung: Herakles, der im Olymp Einzug hält, belohnt für seine Mühen - er hat dabei jedoch, wie wohl ins Auge fällt, gar nichts mit Kunst zu tun. Achill, den Chiron das Saitenspiel gelehrt hat, und den die Vasenbilder oft im Brettspiel mit Ajas zeigen, eignet sich anders zu dem Spiel der Weißen Insel: er ist keine Symbolfigur für den allgemein-menschlichen Aufstieg. Die Kunst als „letzte metaphysische Tätigkeit”, der Schatten als das Apollinische (im Traum sichtbar) in Benns Antwort an Nietzsche in unserem Gedichtzyklus: das letzte Wort ist Paris, das Verhängnis, der Mann der Schönheit, und nicht der dorische Herakles.

In dieser Form tritt uns das Verharren vor dem Unvereinbaren in den Blick: nicht als Bekenntnis des Autors Gottfried Benn, sondern als Werk. Das Gebilde, das man, nach Benns Lieblingsausdruck, zur Hand nehmen, beschauen und umdrehen kann, die poetische Komposition in ihrer Eigenart deutet gerade das Zusammengehören des Unvereinbaren: im Nebeneinanderlegen der Gedichte liegt die Schönheit, die sich bezwingend manifestiert. Hierin beruht zugleich Nähe und Ferne zur Weimarer Klassik, als Verhalten zur Antike: nicht vermittelt über humanistische Kontinuität (sei auch immer ein „Rest” davon spürbar, wie Ausleger gerne feststellen), sondern in der Diskontinuität selbst, über den Abgrund des Bruches, das 19. Jahrhundert und die Katastrophen des 20. hinüber, antwortet die Poesie. Sie beschwört keine dorische, sondern eine äolische Welt des fünften Jahrhunderts. Derart wird übrigens dieses fünfte, das sophokleische Jahrhundert der Griechen aus dem bloß kulturoptimistischen Blickwinkel (als Eingangspforte zu der durch uns selbst verkörperten Höhenwelt) 
herausgesetzt und zugleich als Umschlagspunkt des Archaischen und Modernen erkannt. Dies ist, in Abhebung vom Evolutionismus des neunzehnten, die spezifische Modernität des zwanzigsten Jahrhunderts: Nietzsche, und zwar ein gerade von jenem, Benn eher peinlichen Teilaspekt des Züchtungs-Optimismus unabhängig gedachter Nietzsche, bleibt hier der Orientierungspunkt. Ihm respondiert die aus der expressionistischen Haltung erwachsene Poesie der „Klassik II”: ist allenfalls etwas an ihrem Werk klassisch, so gerade dies, daß in der geschichtlichen Lage des Verharrens vor dem Unvereinbaren nichts anderes als die Selbständigkeit der Teile, das heißt ihre nicht-offenbare Harmonie, zur Sprachgestalt kommt.

Daß die Gedichte, nicht anders als in ihrem Inneren die Verse und ebenso die einzelnen Worte, nur eben im „parataktischen” Nebeneinander-Legen der Gebilde, einander zu antworten und $\mathrm{zu}$ singen beginnen: das bedeutet die Montage jener Worte und Substantive, deren Flug nach einem Wort Benns Jahrtausende entfallen. Die Trance aber, daß es diese Wirklichkeit nicht gebe (Benn teilt sie mit Tiecks William Lovell und mit Paul Valéry), nämlich als von mechanischer Kausalität und linearer Zeit-Evolution determinierte Wirklichkeit ${ }^{18}$, ist darum wohl weniger ein Ausweis von Realitätsverlust, als daß sie, Benns Anspruch übrigens legitimierend, einem Denken entspricht, wie wir es bei Werner Heisenberg (dem notorischen „Irrationalisten” der Weimarer Republik) oder Wolfgang Pauli finden, wonach der Mensch beim Ergreifen von Wirklichem nur noch "sich selbst” begegnet. Wirklichkeitsverlust ist dergestalt, wie eingangs gesagt, eigenes Thema und zumal Formgesetz der Gestaltung Bennscher Poesie, nicht erliegt sie jenem..$^{19}$ Der durch den Gegensatz von Halluzination und Konstruktion bestimmte Kunstcharakter impliziert jene Zerstörung des konventionellen „Ich” des 19. Jahrhunderts, den man, ohne hier nach Denkern wie Ernst Mach fragen zu können, bei Benns Freund Carl Einstein 1932 gefaßt findet: „Die Lehre vom einseitigen Ich wird durch die Lehre vom Verschwinden des Ichs erledigt... und die Lehre von der Einheit des Wirklichen wird durch die Dialektik von der Pluralität des Wirklichen aufgehoben"; mit der Konsequenz: „Für uns gelten halluzinatorische, einfache Ideen, Entrationalisierung und Umgestaltung des Menschen, seines Geistes, seiner Sprache. Besonders durch das Halluzinatorische werden wir $\mathrm{zu}$ den kollektiven Kräften vorstoßen." ${ }^{20}$ Umgriffen wird das lineare Ich von dem "geologischen" Schichten-Ich der Poesie: die alten Dinge, wie sie diesem erscheinen, Substantive wie „Eleusis” wirken darum diesseits der historistischen Vergegenständlichung. Das ist es, was aus Quellen wie Rohdes Psyche entsteht - und das Gedicht Meer- und Wandersagen, das Benn 1925 Carl Einstein gewidmet hat, könnte fast wie eine Poetologie gelesen werden: „unbewegter Raum, / keine Einzeldinge ragen / in den Südseetraum." ${ }^{21}$ Aber man darf hinzufügen, daß die beiden Dichter hier unübersehbar an die Lehren Rimbauds anknüpfen: Arthur Rimbauds, der ja in seiner Jugend lateinische Verse gedichtet hatte. 


\section{NOTES}

1. Vgl. zum Beispiel Jean-Michel Palmier, „Préface”, in G. Benn, Double Vie, tr. A. Vialatte, Paris, Minuit, (1954), 1981, p. XXXIX. Der sich anschließende Vorwurf des „Geschichtshasses”, der sich sehr wohl auf Formulierungen des Dichters berufen kann, leidet an der irrtümlichen Gleichsetzung von Geschichte und Evolutions-Modell: es ist kaum ein Wunder, daß man von daher den eminent geschichtlichen Charakter dieser Poesie nicht recht zu fassen bekommt. Zum Zeithorizont vgl. auch das bei Ulrich Fröschle, „Die Kyklen der Kykliker. Über die Wiederkehr der ,Wiederkehr' bei F. G. Jünger”, in B. Gruber, Hg., Erfahrung und System. Mystik und Esoterik in der Literatur der Moderne, Opladen, Westdeutscher Verlag, 1997, p. 204-225, besonders 221 f., dargestellte Material.

2. Friedrich-Wilhelm Wodtke, Die Antike im Werk Gottfried Benns, Wiesbaden, Limes, 1963. Allgemein vgl. Helene Homeyer, „Gottfried Benn und die Antike”, in B. Hillebrand, Hg., Gottfried Benn, Darmstadt, Wissenschaftliche Buchgesellschaft, 1979, p. 83-96 (die von 1960 stammende Arbeit ist durch nichts ersetzt); ferner einiges bei Regina Weber, „Gnostische Elemente im Werk Gottfried Benns", in H. A. Glaser, Hg., Gottfried Benn 1886-1956, Frankfurt u. a. , Lang, 1989, p. 129-150.

3. Gottfried Benn, „Dorische Welt”, Gesammelte Werke in acht Bänden, hg. von D. Wellershoff, Wiesbaden, Limes, 1968, vol. 3, p. 848. - Die weitere Konsequenz einer solchen Wendung gegen Nietzsche - wäre dann für die Erfassung der deutschen Gegenwart eine Parallele oder ein Chiasmus zu den Griechen denken? - soll hier nicht weiter verfolgt werden.

4. Weiter differenzierend hierzu Beda Allemann, Gottfried Benn: das Problem der Geschichte, Pfullingen, Neske, 1963.

5. Eine Aufzählung sonst im Gedicht fehlender Elemente des 5. Jahrhunderts (weder Perserkriege noch Tragödie, weder Philosophie noch Statuen und Tempel, etc.) bei Edgar Lohner, Passion und Intellekt. Die Lyrik Gottfried Benns (1961), Frankfurt a. M., Fischer, 1986, p. 214 f.

6. Werke, 1, 153.

7. Vgl. beispielsweise Wodtke, op. cit., p. 83.

8. Werke, $1,198$.

9. Vgl. den schönen Nachweis von Klaus Gerth, „Absolute Dichtung?” in Gottfried Benn (o. Anm. 2), p. 253-256.

10. 7.-8. Auflage, Tübingen, Mohr, 1921. Ein solcher Umgang mit der nächtigen Welt von Tod und Jenseits bei den Griechen wirkte damals wohl wie Irrationalismus, ein Zeichen jener romantischen „Sympathie mit dem Tode”, der bekanntlich Thomas Mann das deutsche Unheil zuschrieb; er war, mit einem heutigen Forscher zu reden, „incomprehensible to most 20th-cent. scholars as a consequence of modern ethnocentrism and death-avoidance" (Herbert Hoffmann, Sotades: Symbols of Immortality on Greek Vases, Oxford, Clarendon Press, 1997, p. 56).

11. Wodtke, op. cit., p. 83-96; vgl. Lohner, op. cit., p. $231 \mathrm{f}$.

12. Werke, 1, $201 \mathrm{f}$.

13. Gewisse Lapsus Benns, lehrreich in ihrer Art, werden oft von Kommentatoren diskret übergangen; dazu gehört die falsche Intonation des Plurals „Plutonien” (von „plutonium”, bei Rohde von Benn verkannt), wie anderwärts im Gedicht Orpheus“ Tod die „Haune” („Haun” nicht erkannt als Plural von „Haue” in der Ovid-Übersetzung von Voß); Doste und weißer Thymian sind bei Rohde dasselbe. Die anachronistischen Magnolien zeigen nachher die höhere Wahrheit der poetischen Lizenz.

14. Vgl. Lohner, op. cit., p. 231 f.; Wodtke, op. cit., p. 96.

15. Heraklit, fr. B 93 D.-K. 
16. Nicht weit ist der Gedanke an Goethes Helena (die Insel Leuke erscheint in einem FaustParalipomenon).

17. Nem. 4,49 .

18. Determination, der naheliegender Weise eine solche Denkfigur wie „progressive Zerebration” selbst zugehört: manches mehr wäre davon zu sagen.

19. Ähnlich ist die These von Silvio Vietta, „Gottfried Benns Subjektkritik und sein politischer Fehlschritt”, in Gottfried Benn, 1886 bis 1956, op. cit., p. 229-242.

20. Carl Einstein, Gesammelte Werke, hg. von E. Nef, Wiesbaden, Limes, 1962, p.145. Zur Bedeutung von Benns Freundschaft mit Einstein (1933 gingen beide Autoren bekanntlich in diametral verschiedene Richtungen) ist in politischer wie literarischer Hinsicht viel Treffendes gesagt bei Rainer Rumold, Gottfried Benn und der Expressionismus, Königstein, Scriptor, 1982, p. 64 und passim; für Erwägungen zu „Halluzination und Konstruktion” dürfte die Präsenz des Wölfflin-Schülers und Autors von Negerplastik und Braque in Benns Werk auch heute noch nicht zulänglich ermessen sein.

21. Werke, 1, 66.

\section{RÉSUMÉS}

On considère souvent qu'à la racine de la notoire tyranny of Greece over Germany, sous sa forme ultérieure de nietzschéanisme anti-moderniste, se trouve cet "irrationnalisme » qui se laisse aller au pur et simple dionysiaque : comme l'incarnerait de manière exemplaire, en préalable de son erreur politique de 1933, la poésie nihiliste de Gottfried Benn ; à l'opposé de quoi, une foi en la raison et la mesure apolliniennes, selon l'espoir d'un Thomas Mann, aurait été apte à modifier la voie fatale que devait prendre l'esprit allemand. Quant à cela, il convient cependant de préciser d'abord que Benn, en 1933, érige un credo résolument anti-dionysiaque, préconisant le «monde dorique »; c'est ce constat qui pourvoit ensuite l'arrière-plan d'un regard sur le cycle lyrique V.Jahrhundert. S'y manifestent des tensions paradoxales : l'absence totale de références «doriques » dans ces poèmes permettra ainsi de prendre la mesure de la réponse bennienne au classique schillerien, en ce qui regarde la pensée du «jeu» tout autant que la constitution poétique de l'œuvre cyclique elle-même. Reste enfin le postulat, pour la compréhension de cette poésie faite de «hallucination et construction », d'une analyse suffisante du rapport entre Benn et son ami Carl Einstein.

"Irrationalism", a fatally anti-modern devotion to the Dionysiac, has often been identified as a main component of the notorious "tyranny of Greece over Germany”. In its later shape as Nietzsche-imitation, it seems perfectly embodied in Gottfried Benn.s poetry of the twenties, as the very precondition of this poet.s political aberration in 1933 - when, by virtue of a turn towards Apolline measure, the German predicament might still have found a less gruesome issue (such was at least the hope of Thomas Mann). In view of this, it should be observed in the first place that Benn.s 1933 credo is in fact a resolutely anti-Dionysiac statement of the "Doric universe"; against this background, then, an exegesis of the lyric cycle V. Jahrhundert may be attempted. Tensions become visible, bordering on paradox: the absence of „Doric" references in the poems leads to a reflection on Benn.s response to Schillerian classicism, engaging the idea of "play" no less than the artistic shape of the cyclical work itself. As it turns out, for an adequate 
perception of this poetry marked by „hallucination and construction” to come about, one must be aware of Benn.s relationship to his friend, Carl Einstein.

Als Hauptmerkmal der tyranny of Greece over Germany in ihrer späteren Ausprägung als NietzscheNachfolge gilt oft der „Irrationalismus” einer verhängnisvoll antimodernen Hingabe an das Dionysische, wie sie auch der Dichter Gottfried Benn in der rauschhaft-ichfeindlichen Poesie der zwanziger Jahre, präzise als Bedingung seines politischen Irrgangs von 1933, beispielhaft zu verkörpern scheint: mit einem Bekenntnis zur Vernunft apollinischen Maßes hätte sich demnach vielleicht (so hoffte es Thomas Mann) die deutsche Fatalität in andere Bahnen lenken lassen. Demgegenüber ist zunächst festzuhalten, daß Benn 1933 vielmehr ein ausgesprochen antidionysisches Credo der „dorischen Welt” aufstellt; vor dessen Hintergrund wird alsdann der lyrische Zyklus V. Jahrhundert ausgelegt. Paradox anmutende Spannungen bleiben nicht aus: an die Beobachtung des völligen Fehlens „dorischer” Bezüge in der Dichtung knüpft sich eine Erwägung von Benns Antwort auf die Schillersche Klassik, im Gedanken des „Spiels” nicht anders als in der Gestalt des zyklischen Werkes selbst. Zu fordern für das Verständnis dieser Poesie von „Halluzination und Konstruktion” bliebe eine zulängliche Analyse von Benns Verhältnis zu seinem Freund Carl Einstein.

INDEX

Mots-clés : poésie

oeuvrecitee Fünftes Jahrhundert

\section{AUTEURS}

HOLGER SCHMID

Université Charles-de-Gaulle - Lille 3 